\title{
Nonorthogonal Multiple Access for Next-Generation Mobile Networks: A Technical Aspect for Research Direction
}

\author{
Muhammad Hussain (D) and Haroon Rasheed \\ Electrical Engineering Department, Bahria University Karachi Campus, Karachi 75260, Pakistan \\ Correspondence should be addressed to Muhammad Hussain; engr.m.hussain.bukc@bahria.edu.pk
}

Received 22 March 2020; Revised 14 July 2020; Accepted 29 October 2020; Published 30 November 2020

Academic Editor: Farman Ullah

Copyright (c) 2020 Muhammad Hussain and Haroon Rasheed. This is an open access article distributed under the Creative Commons Attribution License, which permits unrestricted use, distribution, and reproduction in any medium, provided the original work is properly cited.

\begin{abstract}
5G mobile communications offer several benefits, which include providing extremely low latency, very high data rates, significant improvement in the number of users, and increase in base station capacity and perceived quality of service. This may be achieved at the cost of an increased receiver complexity by nonorthogonal access of users. Nonorthogonal multiple access (NOMA) is one of the capable contenders to achieve the vision of $5 \mathrm{G}$ wireless communications. Supporting a higher number of users than available orthogonal resources is the key feather of NOMA. In this article, the basic principle of NOMA has been reviewed and compared with other orthogonal multiple access (OMA). A comprehensive survey is presented in the latest NOMA scheme. The distinguished NOMA schemes design principle features, and recent deployments are discussed. Furthermore, the performance is compared in terms of the bit error rate, system capacity, and energy efficiency. The performance results show that NOMA can achieve the required goals, in terms of the user data rate, system capacity, interference cancellation scheme, and reception complexity.
\end{abstract}

\section{Introduction}

Multiple access schemes have been a landmark technology from $1 \mathrm{G}$ to $4 \mathrm{G}$ for the growth of mobile communications. As a design aspect, these multiple access technologies are mostly from the orthogonal multiple access (OMA) category; they are in the time domain, code domain, frequency domain, and time-frequency domain. OMA can easily detect the user information signal by utilizing a simple receiver. However, the entire number of users that the system can accommodate is firmly restricted by the number of available orthogonal resources. Also, the system requirements for synchronization are highly limited in order to guarantee the orthogonality of resource allocation among users. Therefore, it is very difficult for OMA to meet the data rate and other requirements of the next-generation mobile network. The $5 \mathrm{G}$ structure demands an innovative multiple access scheme to counter this challenge and recently proposed nonorthogo- nal multiple access (NOMA) technology which is accepted as a $5 \mathrm{G}$ multiple access scheme $[1,2]$.

Within the common physical layer using the code domain or power domain multiple access, NOMA permits numerous users to utilize frequency and time resources [3]. In recent times, various NOMA topologies have received a lot of attention due to attractive features. We can generally categorize into two types. These types are code domain multiple access and the power domain multiple access. NOMA achieved its goals by a combination of multiple access techniques like sparse code multiple access (SCMA) [4], multiuser shared access (MUSA) [5] with Low-Density Spreading (LDS) [6], and Pattern Division Multiple Access (PDMA) [7].

1.1. Motivation. In September 2014, the 3rd generation partnership project (3GPP) started the study on NOMA in Release 14 (Rel-14). NOMA may be combined with upcoming wireless communication systems in order to achieve the 
requirements, including massive connectivity, high spectral and energy efficiency, significant achievable data rate, low latency, exceptional user fairness, large throughput, ultrahigh reliability, and upholding different quality of services (QoS).

Some previous impressive survey work on NOMA is followed. In [8], the transceiver block diagram of each category of NOMA is explained by the authors, regarding detailed key features, basic principles, and algorithms of the transmission-reception. In [9], characteristics and working principles of different NOMA schemes are summarized by the authors. In [10], NOMA schemes are compared and analysed by the authors. The authors focus on the future research directions of NOMA, prototype development, recent progress, standardization, and challenges. In [11], some promising nonorthogonal schemes were discussed which include sparse code multiple access (SCMA), Power Domain Nonorthogonal Multiple Access (PD-NOMA), Pattern Division Multiple Access (PDMA), multiuser shared access (MUSA), and some key modern waveforms including Generalized Frequency Division Multiplexing (GFDM), Universal Filtered Multicarrier (UFMC), and filter bank-based multicarrier (FBMC). The authors provided a future research path for $5 \mathrm{G}$ waveform and multiple access schemes by comparing and analysing the characteristics of these technologies.

However, in [8], achievable sum rate performance was presented, based on average mutual information rather than actual theoretical analysis. In [9], without mathematical justification, the performance of the NOMA schemes is assessed. In $[10,11]$, performance evaluation has not been examined by the authors. Furthermore, most of the previous work may just focus on one scheme, and no comprehensive work has been published to examine the performance of major NOMA schemes.

The objective of this research is to fill in the gap by presenting the basic principles, key features, and recent application of major categories of NOMA. Moreover, we present actual theoretical analysis and mathematical justification of the NOMA schemes. The major contributions are summarized as follows.

1.2. Contribution. In this article, a comprehensive and comparative survey on NOMA is presented.

(i) The survey includes different popular categories of NOMA, their basic model, working principles, technical aspect, key performance indicators (KPIs), advantages, and disadvantages

(ii) The article presents the state-of-the-art review of NOMA in enabling the $5 \mathrm{G}$ network, the applicability aspect of each category of the NOMA scheme, and the associated enablers

(iii) Moreover, in this article, we present important and recent deployments, potential challenges, and future trend work for researchers in the field of NOMA

(iv) The survey also includes the performance comparison of major categories of NOMA prototype in terms of achievable data rate, system capacity, energy efficiency, and bit error rate with mathematical justifications

Furthermore, this article is planned as follows: Section 2 is a recall history of mobile communication and their technology aspect. Section3 explains and investigates important nonorthogonal multiple access schemes with their principle of implementation, followed by a review of every scheme's key features and advantages and disadvantages. A summary of the NOMA scheme is presented in Section 4, and discussion of the results is presented in Section 5. Section 6 presents a review of recent developments in NOMA schemes, Section 7 presents the future research challenges of NOMA, and in Section 8, a conclusion is made.

\section{Background}

In the third generation mobile system, the Wideband Code Division Multiple Access (WCDMA) scheme was launched. As a result, movies can be transmitted due to improved speed of data communication. Furthermore, 3G presented an improved technology, i.e., High-Speed Packet Access (HSPA) and HSPA+ (3.5G), with which the user data experience was improved. However, in comparison to $\mathrm{Wi}-\mathrm{Fi}$ and wireless LANs, high data rate applications like streaming of moving images were slower. Today, network operators provide services of $4 \mathrm{G}$ networks based on Long-Term Evolution (LTE). The achievable communication speed rises up to 5 to 6 times in comparison to $3 \mathrm{G}$, and data throughput is also expressively enhanced in LTE than HSPA+. In LTEAdvanced (LTE-A), the available bandwidth is twice as LTE; therefore, several $4 \mathrm{G}$ network service providers are also transferred to LTE-Advanced (4.5G). With LTE and LTEAdvanced, communication technology has improved, at a level close to $\mathrm{Wi}-\mathrm{Fi}$ with respect to user data experience. $4 \mathrm{G}$ network and LTE and LTE-A technology are saturated in terms of further improvement. The wireless data requirement is increasing day by day. Therefore, there is a need for new technology to speed up data access. However, for wireless communication, improvement in the data capacity and the data transmission rate is essential. Therefore, for the mobile Internet extension and modernization, researchers all over the world started investigating ways to improve data capacity and data transfer rates.

Meanwhile, from the beginning of digital communications in the 1990s, cellular phone technology has been on the track in terms of progress, focused on increasing the data rate and capacity. In the current world communication trends, mobile Internet and video calling have become a reality, and its new version has been launched, i.e., 5G mobile communication. Now, at any emergency condition such as online medical imaging or smart vehicles in congestion, more data needs to be delivered to the specific user. Thus, $5 \mathrm{G}$ networks will respond accordingly. Researchers also recognize $5 \mathrm{G}$ as an opportunity to redefine not only the network enable connecting a wide variety of new devices but also the networks that realize exceptional data rates. The next version of $5 \mathrm{G}$ wireless mobile technology is $6 \mathrm{G}$, which means 6 th generation wireless mobile technology. Satellite networks 
for global coverage will be efficiently used in $6 \mathrm{G}$ which was not used before [12]. The $6 \mathrm{G}$ wireless mobile technology maximizes data throughput and improves system performance. The $6 \mathrm{G}$ technology is responsible for more data transfer and data security. It also increases data configuration choices. In 6G technology, devices connected to the Internet by using wireless broadband receive $10 \mathrm{~GB}$ or even more data speed. $6 \mathrm{G}$ is a satellite-based network; roaming and handover from one satellite to another satellite are still an issue which will be solved soon. The combination of fiber optics and the latest radio technology is used in $6 \mathrm{G}$, to provide a very fast data experience. The $6 \mathrm{G}$ wireless mobile technology will change the way of thinking about wireless communication and will perform beyond the expectation of the users [12]. Moreover, this performance depends on technology use in next-generation networks.

Numerous proposals have been presented by researchers to establish the performance of NOMA in both downlink and uplink. The basic principle of downlink NOMA is presented in [13], power division is used for multiple user access at BS, and SIC is used for signal detection at the receiver. In [14], researchers proposed a two-user model for NOMA. Researchers presented link-level simulations and systemlevel simulations for the NOMA downlink system. Results provided in [14] showed that NOMA performance is better than OMA in terms of overall system throughput and individual user throughputs. The authors in [14] derived the closed-form expressions for outage probability and ergodic sum rate for the NOMA downlink system. In [15], to find the effect of user pairing for the two-user model of the NOMA system, the authors employed statistically allocated transmit powers among NOMA users. Moreover, the authors proposed fixed and opportunistic user pairing schemes. In [16], the authors consider the consequence of power allocation on fairness. To ensure that users are getting an equal share of system resources, the fairness index should be close to 1 . The authors proposed a power allocation scheme to maintain the fairness index. In [17], the authors used the concept of user pairing; the authors paired strong channel users along with weak channel users for the cooperative NOMA system through imperfect CSI and perfect CSI feedback. The authors in [18] presented NOMA-aided precoded spatial modulation (NOMA-PSM) in which researchers combined NOMA with Multiple Input Multiple Output (MIMO). Researchers also presented a comparison with OMA in terms of implementation cost, multiuser interference, spectral efficiency, and performance gain of the system. In [19], the authors proposed full-duplex NOMA relayingbased Device-to-Device (D2D) communication. The authors in [19] proposed the solution for the D2D power allocating problem by presenting a linear fractional programmingbased power allocation scheme.

The basic principle of uplink NOMA is presented in [20], the SIC signal detection scheme is utilized at BS, and the power control scheme is used at the user side. The authors investigated the challenges of joint power allocation and subcarrier assignment, and the authors designed a suboptimal solution to increase the sum rate of the NOMA cluster. In [21], the researchers derived the closed-form expressions for outage probability and system capacity for the two-user model of the NOMA uplink system. The researchers investigated the static powers for several users and recognized that a user could be in outage without proper selection of the required data rate. In [22], for the uplink PD-NOMA system, the authors presented an adaptive power control scheme which is based on Evolutionary Game Theory (EGT). To enhance users' throughput or payoffs, the proposed power control scheme allows users to adaptively adjust their transmit power level. SIC is used for signal detection at the receiver. In [23], researchers provided the advantages and challenges of NOMA as a contender scheme in dense networks. The authors compared the performance of NOMA in UL systems. To compare the performance of WSMAbased NOMA and MU-MIMO, researchers presented linklevel evaluation results. In [24], the authors provided a foundation to investigate multicell uplink NOMA systems. The authors considered the coverage probability of a NOMA user with high interference at the BS due to a large number of cochannel NOMA transmitting users. The authors in [24] provided closed-form expression of the rate of coverage by characterizing the Laplace transform of the intercluster interference in different SIC scenarios. Afterward, the authors characterized the Laplace transform of the intercluster interference through distance distribution from geometric probability. To evaluate the benefits of NOMA, in 2018, 3 GPP considered NOMA as a research icon and provided guidelines to support NOMA, in comparison to the OMA [25]. Table 1 summarizes the review of nonorthogonal multiple access.

\section{Nonorthogonal Multiple Access}

NOMA is a diverse multiple user access scheme with respect to other established and existing multiple access schemes, i.e., orthogonal multiple access. At the transmitter side, NOMA deliberately introduces intercell and/or intracell interference; therefore, it can utilize nonorthogonal transmission. At the receiver side, successive interference cancellation (SIC) technique is used to decode the desired signal. In comparison with orthogonal multiple access, the complexity of the receiver is increased, but better spectral efficiency can be achieved. Therefore, the fundamental concept of nonorthogonal access is to utilize a receiver with a complex design in trade-off for high spectral efficiency. Therefore, the enhancement in chip processing technology makes the nonorthogonal access scheme possible.

3.1. Power Domain Multiple Access. The NOMA scheme consists of two key technologies. One is power domain NOMA (PD-NOMA), which utilizes efficiently the SIC scheme in order to perform multiuser detection. SIC is a famous physical layer interference cancellation scheme which is used to receive two or more users' signals simultaneously [46]. SIC is sufficiently used in comparison to the existing scheme which causes degradation of the signal. In the SIC scheme, the strongest signals are subtracted from the received combined signal one after another by the SIC receiver; finally, the SIC receiver extracts the desired signal. It is a gradual 
TABLE 1: State-of-the-art review of nonorthogonal multiple access.

\begin{tabular}{cc}
\hline Ref. & Objective \\
\hline & $\begin{array}{c}\text { Improve reliable detection, } \\
\text { maximum diversity gain, } \\
\text { and reduce system } \\
\text { complexity. }\end{array}$
\end{tabular}

Achieve the fairness performance of the NOMA scheme better than TDMA under perfect and average CSI.

Further improve the outage performance of MIMO-NOMA.

$$
\begin{gathered}
\text { Resource allocation algorithm } \\
\text { design }
\end{gathered}
$$

for multicarrier NOMA systems.

[29] Multiple half-duplex uplink and downlink users simultaneously served by a full-duplex base station.

For the downlink NOMA system, optimized power allocation and subchannel assignment to increase energy efficiency.

Improve the link-level performance

[31] of SCMA in highly overloaded scenarios.

Maximize the mutual information in sparse code multiple access (SCMA).

Substantially minimize the hurdles

[33] of the message passing algorithm

(MPA) scheme.

Reduce the decoding hurdles of the current message passing algorithm.

Minimizing the hurdles of the SCMA decoding.

Maximize the sum rate subject to

[36] QoS and system-level constraints like power constraints.

For random signature selection, [37] allowed grant-free transmission to achieve high overloading.

For the paired users, optimized the modulated symbol mapping.

Solution approach

The highest diversity gain with minimum outage probability achieved by cooperative

PD-NOMA. User pairing is used as a promising solution to reduce system complexity.

Investigated power allocation techniques that ensure fairness by formulating the research problems as nonconvex optimization.

Improvement achieved by implementing detection and precoding matrices for MIMO-NOMA.

An algorithm is designed for multiple half-duplex uplink and downlink users simultaneously served by a full-duplex base station. Used weighted sum system throughput maximization from the solution of a nonconvex optimization problem.

For subchannel multiplexed users, a low-complexity suboptimal algorithm is presented, which comprises power proportional factor determination and energy-efficient subchannel assignment.

Proposed an iterative multiuser SCMA receiver by employing channel coding which uses the coding gain and diversity gain.

Maximize the mutual information between continuous output and discrete input using an iterative codebook optimization algorithm.

For uplink SCMA systems, a shuffled-message passing algorithm (S-MPA) scheme is proposed, based on a serial message update strategy.

Based on list sphere decoding (LSD), a low-complexity decoding algorithm is proposed. The LSD only works with signals inside a hypersphere by evading the extensive search for all possible hypotheses. Proposed a Monte Carlo Markov Chain- (MCMC-) based SCMA decoder. Benefiting from the linearly increasing complexity of the MCMC method.

Multiple users utilized the same SCMA codebook, and for user signal nonorthogonality, the PD-NOMA scheme is utilized.

Introduced a blind multiple user detection for MUSA systems by using a special blind detection algorithm.

Performance of MUSA with SIC has been considered by using mirror constellation bit error ratio (BER).
Single carrier power domain
Co-PD-NOMA

Single carrier power domain

PD-NOMA

Single carrier power domain

MIMO-NOMA

Multicarrier power domain

MC-NOMA

Single carrier power domain

PD-NOMA

Multicarrier code domain

SCMA

Multicarrier code domain

SCMA

Multicarrier code domain

S-MPA

Multicarrier code domain

LDS

Multicarrier code domain

MCMC

Power \& code domain

PD-SCMA

Single carrier code domain

MUSA

Single carrier code domain

MUSA 
TABLe 1: Continued.

\begin{tabular}{|c|c|c|c|c|}
\hline Ref. & Objective & Solution approach & Category & Tech. \\
\hline [39] & $\begin{array}{l}\text { A family of short length complex } \\
\text { sequences is selected to permit } \\
\text { an easy multiuser interference } \\
\text { cancellation. }\end{array}$ & $\begin{array}{l}\text { Successive/parallel interference cancellation } \\
\text { with minimum mean square error } \\
\text { (MMSE-SIC/PIC) has been investigated } \\
\text { for appropriate MUSA receivers. }\end{array}$ & Single carrier code domain & $\begin{array}{l}\text { MMSE- } \\
\text { SIC/PIC }\end{array}$ \\
\hline [40] & $\begin{array}{l}\text { Increase user overloading and } \\
\text { minimize multiuser interference. }\end{array}$ & $\begin{array}{c}\text { Enlarge the pool of the spreading } \\
\text { sequences by using nonorthogonal } \\
\text { dense spreading sequence to increase } \\
\text { user overloading and reduce } \\
\text { multiuser interference. }\end{array}$ & Single carrier code domain & MUSA \\
\hline [41] & $\begin{array}{c}\text { To further enlarge the coverage } \\
\text { area and improve transmission } \\
\text { reliability. }\end{array}$ & $\begin{array}{l}\text { With forward relay and half-duplex } \\
\text { decode, an uplink cooperative PDMA } \\
\text { (co-PDMA) scheme is suggested. }\end{array}$ & Single carrier code domain & Co-PDMA \\
\hline$[42]$ & $\begin{array}{l}\text { Increase the performance of PDMA } \\
\text { uplink system by using diversity } \\
\text { gains and coding potentials. }\end{array}$ & $\begin{array}{l}\text { By using diversity gains and coding } \\
\text { potentials, an iterative detection and } \\
\text { decoding (IDD) algorithm is } \\
\text { developed for an advanced } \\
\text { PDMA receiver. }\end{array}$ & Single carrier code domain & IDD \\
\hline [43] & $\begin{array}{l}\text { Using the cyclic redundancy check } \\
\text { (CRC) to avoid the error } \\
\text { propagation. }\end{array}$ & $\begin{array}{l}\text { Based on the MMSE channel decoding } \\
\text { and detection, a novel iterative } \\
\text { decoding and detection algorithm is } \\
\text { proposed, called the SIC iterative } \\
\text { processing algorithm. }\end{array}$ & Single carrier code domain & SIC-MMSE \\
\hline$[44]$ & $\begin{array}{l}\text { Proposed the power allocation } \\
\text { and pattern assignment in the } \\
\text { downlink PDMA system. }\end{array}$ & $\begin{array}{l}\text { To optimize the overall throughput } \\
\text { of total users based on the optimum } \\
\text { Iterative Water-Filling (IWF) } \\
\text { algorithm, a joint pattern assignment } \\
\text { and power allocation (JPPA) } \\
\text { scheme is offered. }\end{array}$ & Single carrier code domain & JPPA \& IWF \\
\hline [45] & $\begin{array}{l}\text { Improve security by changing } \\
\text { the signal's identity. }\end{array}$ & $\begin{array}{c}\text { Physical layer security system is } \\
\text { suggested based on constellation } \\
\text { scrambling }(\mathrm{CS}) \text { and multiple parameter } \\
\text { weighted fractional Fourier } \\
\text { transform (MP-WFRFT). }\end{array}$ & Single carrier code domain & MP-WFRFT \\
\hline
\end{tabular}

interference elimination strategy. This type of technique is also used in CDMA to eliminate Multiple Access Interference (MAI). First, the MAI introduced by the user might be eliminated with the help of a signal amplitude recovery process by subtracting the individual user's amplitude one at a time from the received signal. The same process is carried out repetitively to subtract remaining users and to decode the desired signal [47]. Secondly, the PD-NOMA multiplexing scheme uses the power domain technology, that is, power domain multiple access (PDM), which was not used efficiently in previous schemes as used in the PDNOMA scheme. In the power domain, multiplexing nonorthogonality is deliberately introduced. In fact, power dissimilarity among paired users and implementation of SIC within the power domain ensure that user demultiplexing is concurrent. It is different from the other common methods used previously to control power. Also, an algorithm is needed to be used for power distribution at the base station [48].

Figure 1 illustrates the PD-NOMA system with an SIC computation unit. User Equipment (UE) is uniformly distributed in every cell. With different transmitted power of multi- ple users in each subband, the base station (BS) performed downlink transmission for multiple users simultaneously.

Multiple single users can be scheduled at the same time for the same subband by implementing the Proportional Fair (PF) scheduling scheme at BS in the PD-NOMA system. The scheduling procedures for users are described in Figure 2 [49]. First, the BS selects a set of users known as the "NOMA candidate user sets," in which total users cannot exceed $N_{\max }$. The selected user set is prepared by using the total number of possible combinations of users within one single cell. Secondly, for every user set, BS allocates the transmission power by using a power allocation scheme. The scheduling metric for the corresponding user set is estimated on behalf of power assignment ratios. Thirdly, with the help of the maximum scheduling metric, the scheduler decides the candidate user sets on each subband for data transmission. Finally, for every allocated subband, the scheduler estimates equivalent signalto-interference-plus-noise ratios (SINRs) for every single scheduled user. The Coding and Modulation Scheme (CMS) determines the SNR for each user [49].

In PD-NOMA, the total transmit power " $P$ " is divided among multiple users. Let a group of " $k$ " user equipment 


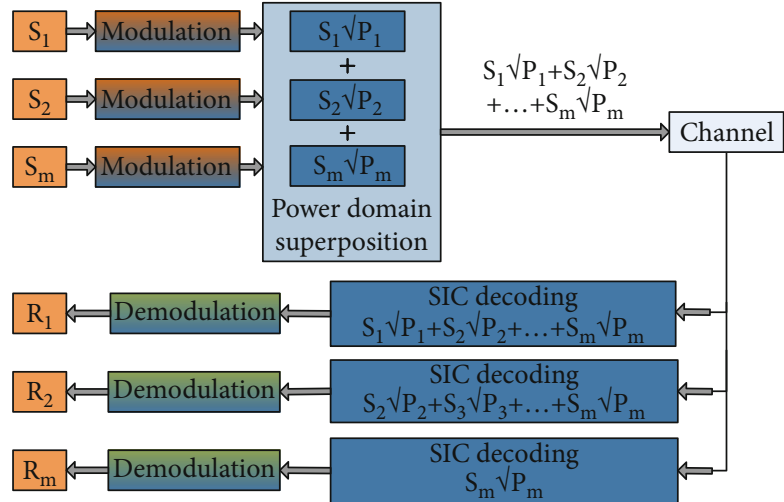

FIgURE 1: PD-NOMA system with SIC.

(UE) be located under the same base station (BS). Therefore, the fraction of power allocated to the $k_{\mathrm{th}}$ user by BS is $p_{k}$, where $p_{k}+\sum_{i=1}^{k-1} p_{i}=P$. A typical NOMA system model is shown in Figure 1 . The received signal at the $k_{\text {th }}$ receiver can be written as

$$
y_{k}=s_{k} \sqrt{p_{k}} g_{k}+\sum_{i=1}^{k-1} \sqrt{p_{i}} g_{k}
$$

where $s_{k} \sqrt{p_{k}} g_{k}$ is the received vector of the $k_{\text {th }}$ user and $\sum_{i=1}^{k-1} \sqrt{p_{i}} g_{k}$ is the interference due to other users.

For PD-NOMA of the downlink system [50], the SINR of the $k_{\text {th }}$ user can be written as

$$
\operatorname{SINR}_{k}=\frac{p_{k}\left|g_{k}\right|^{2}}{N_{0} W+\sum_{i=1}^{k-1} p_{i}\left|g_{k}\right|^{2}} .
$$

Also, throughput for the $k_{\text {th }}$ user can be written as

$$
R_{k}=\mathrm{W} \log _{2}\left(1+\frac{p_{k}\left|g_{k}\right|^{2}}{N_{0} W+\sum_{i=1}^{k-1} p_{i}\left|g_{k}\right|^{2}}\right)
$$

where $p_{k}$ and $p_{i}$ are power allocated to the $k_{\text {th }}$ and $i_{\text {th }}$ users, $g_{k}$ is the channel gain coefficient of the $k_{\text {th }}$ user, $N_{0}$ is the noise density, and $W$ is the bandwidth.

3.2. Sparse Code Multiple Access. By means of a typical NOMA technology, SCMA is conceived as the most promising next-generation multiple access scheme for communication networks. SCMA combines Low-Density Spreading (LDS) and multidimensional modulation (MDM) through the SCMA encoding process [51]. In MDM, the numbers of propagating modes have been scaled to the number of available carrier dimensions, as it is considered coded modulation. For a small set of subcarriers, each user spreads its data via a distinguished LDS. Therefore, more than one user can share each subcarrier because there is no exclusivity in the subcarrier allocation. Compared to the total number of users at every subcarrier, a user will have a relatively small number of interferes [52]. In SCMA uplink scenarios, code- book sets are assigned to every user and users select the random codewords from the dedicated codebook sets. All of the users' codewords are multiplexed and shared at the same orthogonal medium, that is, the OFDM subcarrier, illustrated in Figure 3 [53]. Therefore, the multidimensional codebook plays a crucial role in SCMA systems.

In the SCMA system model, a map is defined as an SCMA encoder in which from $\log _{2}(M)$ to $M$ bits of $K$-dimensional complex codebooks are available. $K$ represents the spreading factor of the system, which is the length of an SCMA codeword. Sparse vectors are a value of $N(N<K)$ which is the nonzero entries of $K$-dimensional complex codewords from the codebook. If $N=2$, two-dimension constellation points can be mapped over $k>2$ resources. A user could be configured with a codebook by using a contention-based multiple access scheme for uplink transmission [54]. A K-dimensional codeword is carefully chosen from the codebook which is used for mapping a user's data bits for transmission on $K$ radio resources (Figure 4 [55]) which are subcarriers of the OFDMA scheme. Each block of SCMA is carried over $K$ number of OFDMA tones.

Let an SCMA uplink system with " $M$ " numbers of users or codebooks, where " $K$ " is the length of the codeword and " $N$ " number of the nonzero elements are present in each codeword. " $d_{m}$ " is the distance between $m_{\text {th }}$ user " $U_{m}$ " and BS. Over $K$ subcarriers, $M$ users are multiplexed. The received signal over all subcarriers $y=\left[y_{1}, y_{2}, y_{3}, \cdots, y_{k}\right]^{T}$ at BS can be written as

$$
y=\sum_{m=1}^{M} \sqrt{\frac{p_{m}}{N}} \operatorname{diag}\left(f_{m}\right) \operatorname{diag}\left(h_{m}\right) x_{m}+w,
$$

where " $P_{m}$ " is the transmission power of user $U_{m} \cdot x_{m}=$ $\left[x_{m 1}, x_{m 2}, x_{m 3}, \cdots, x_{m k}\right]^{T}$ is the codeword or transmit symbols of user $U_{m}$. The channel coefficient vector for user $U_{m}$ is $h_{m}=\left[h_{m 1}, h_{m 2}, h_{m 3}, \cdots, h_{m k}\right]^{T}$.

For the SCMA uplink system [56], the average SNR can be written as

$$
\mathrm{SNR}=\sum_{m=1}^{M} \frac{f_{m k} p_{m}\left|g_{m k}\right|^{2}}{N d_{m}^{\alpha}}
$$

For the SCMA uplink system [56], the average sum rate can be written as

$$
R=\sum_{k=1}^{K} E\left(\log _{2}\left(1+\sum_{m=1}^{M} \frac{f_{m k} p_{m}\left|g_{m k}\right|^{2}}{N d_{m}^{\alpha}}\right)\right)
$$

where $\alpha$ is the path loss exponent, $g_{m k}$ is the channel gain for the $m_{\text {th }}$ user on the $k_{\text {th }}$ subcarrier, $f_{m k}$ is the subcarrier index, and $p_{m}$ is the power of the $m_{\text {th }}$ user.

3.3. Multiuser Shared Access. Multiuser shared access (MUSA) uses the advanced successive interference cancellation (A-SIC) scheme and the advantages of good spreading sequences (SS). In SS, the data bit sequence is encoded per 


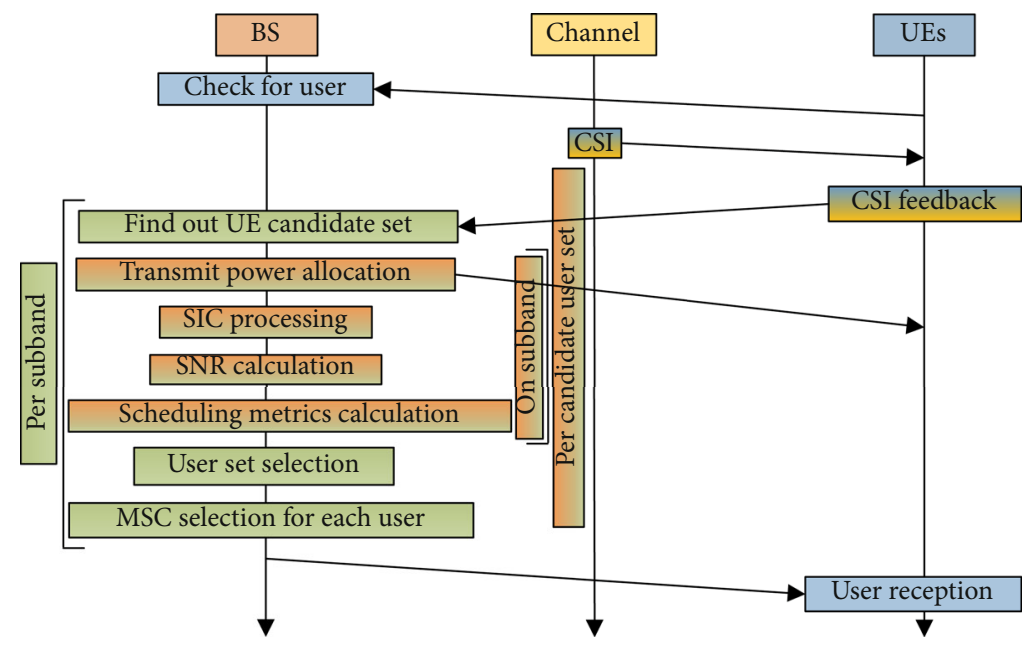

FIgURE 2: Scheduling algorithm for PD-NOMA.

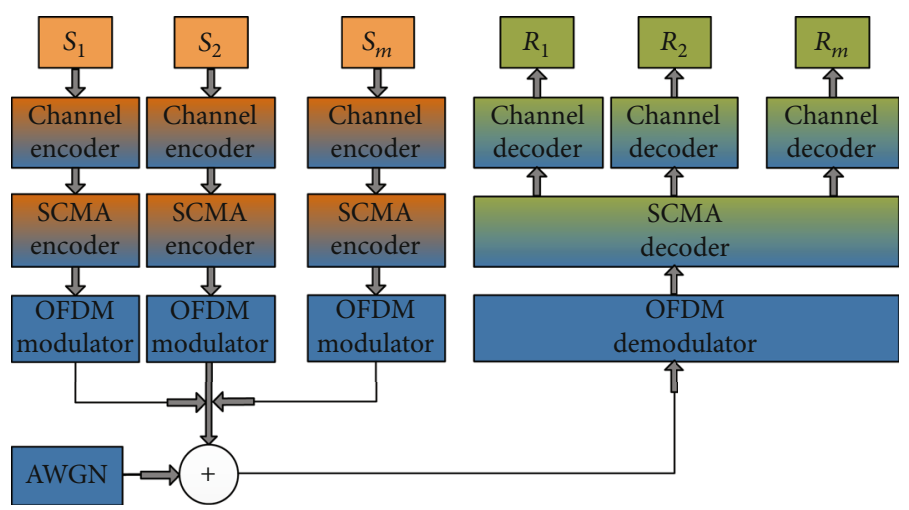

FIGURE 3: SCMA uplink system with $m$ users.

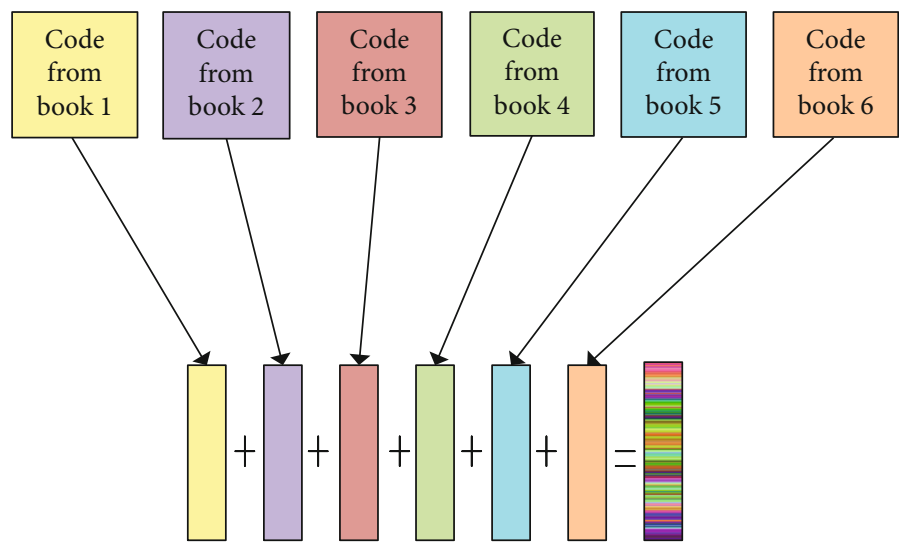

FIGURE 4: SCMA code multiplexing.

codeword. Therefore, at the same time, the identical number of codewords could be encoded by utilizing the encoder. Afterward, the coded bits are permuted (arranged in all possible ways) through random interleaving patterns. If a large number of interleaving patterns are used, the permuted sequence would be statistically independent. The coded sequence is distributed to each subcarrier after modulation on the quadrature amplitude modulation scheme [57].

MUSA uses special spreading sequences, for spreading multiple users' individual data. After that, the user's spread data is overlapped and transmitted. For recovering and demodulating the data of individual users at reception, 


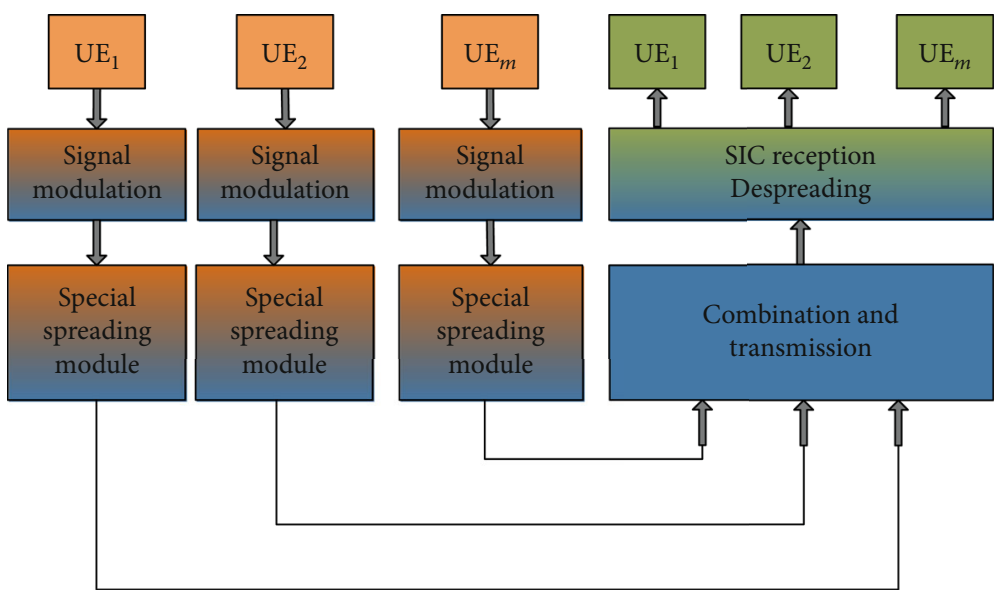

FIgURe 5: Multiuser shared access.

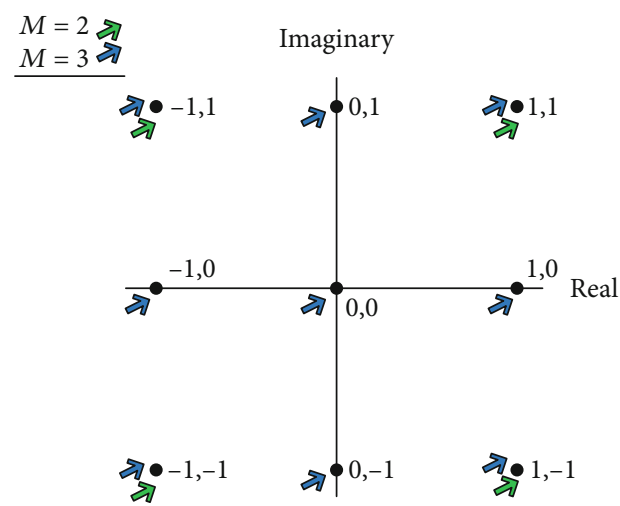

Figure 6: Elements of complex spreading code.

MUSA uses A-SIC receiver. The basic idea is illustrated in Figure 5 [58]. To allow grant-free transmission and maintain a higher overloading factor of users, the nonbinary complex spreading codes at the SIC receiver can be used.

The SIC algorithm is chosen by the SIC receiver to achieve the nonorthogonality between users. It is designed to reduce the power, delay, and complexity if there is large user overloading, and a requirement arises by short spreading codes. A good choice is to use one of the types of the Multicode Complex Domain (MCCD). Due to the design flexibility with the imaginary part and real part, the length of multi-MCCD could be shortened. The sequence with component \pm 1 is a type of complex spreading code that might be created as shown in Figure 6 [58]. Moreover, Figure 6 shows the real and imaginary parts of the code for $M=2$ and $M=3$. Therefore, before normalization, all elements of the complex spreading codes are just elements of the set $\{1-i,-1-i,-1+i, 1+i\}$ because the values of the imaginary part and real part contain 1 and -1 . The maximum number of existing codes is $4^{\mathrm{L}}$ for the code length $L$. In the current scenario, the maximum existing code is 256 for the code length of 4 , which is not sufficient. Therefore, the existing elements of the set which include the imaginary part and real part need to increase, to improve the number of existing codes, which should be $M$-ary with $M>2$.
A preferred selected value of $M$ is 3 ; the sequence with components 0 and \pm 1 is a type of complex spreading code that might be created as shown in Figure 6. Therefore, before normalization, all elements of the complex spreading codes are just elements of the set $\{-1+i,-1+0 i,-1-$ $i, 0-i, 1-i, 0+0 i, 1+0 i, 1+i, 0+i\}$ because the values of the imaginary part and real part contain 0,1 , and -1 , that is, a 3-ary [58]. With the help of the new set, $9^{L}$ codes could be created, which bring considerable improvement in the number of user access.

For multiuser detection at the receiver, SIC is used in MUSA. Linear conjunction of the received signal detects symbols of multiple users. For the linear system, MMSE is used for detecting users. The received signal can be written as

$$
\begin{aligned}
& y=h x+n, \\
& \tilde{x}=h^{-1} y-\tilde{n},
\end{aligned}
$$

where " $x$ " is the composite transmitted signal, " $h$ " is the channel coefficient matrix, and " $n$ " is a complex noise sample of Gaussian noise with zero mean and variance " $\sigma$."

To detect the signal of each user at the receiver, compute the inverse of channel matrix " $h{ }^{-1}$ "; by this inverse, we get the estimated signal " $\tilde{x}$." The MMSE weight matrix can be written as

$$
W_{\mathrm{MMSE}}=\left(h^{H} h+\sigma^{2} I\right)^{-1} h^{H}
$$

where " $I$ " is the identity matrix. Now, we get

$$
\tilde{x}=W_{\text {MMSE }} y \text {. }
$$

The SINR at the $i_{\text {th }}$ antenna of the MUSA uplink system [59] can be formulated as

$$
\operatorname{SINR}_{i}=\frac{E_{x}\left|w_{i} h_{i}\right|^{2}}{E_{x} \sum_{l \neq i}\left|w_{i} h_{l}\right|+\sigma\left|w_{i}\right|^{2}} .
$$



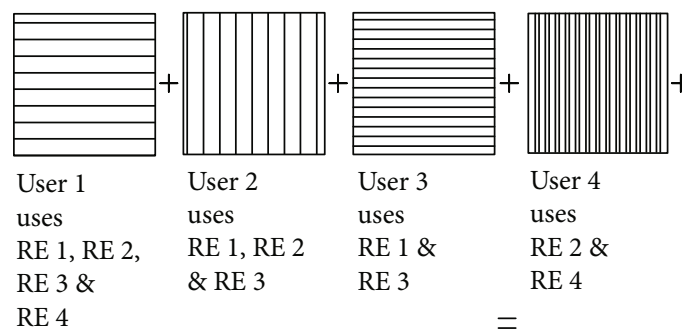

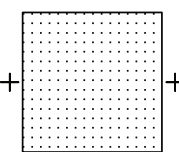

User $5 \quad$ User 6

uses uses

RE $3 \quad$ RE 4 RE 4

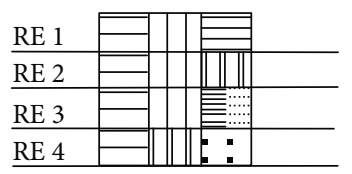

Figure 7: PDMA pattern for 4 REs used by 6 users.

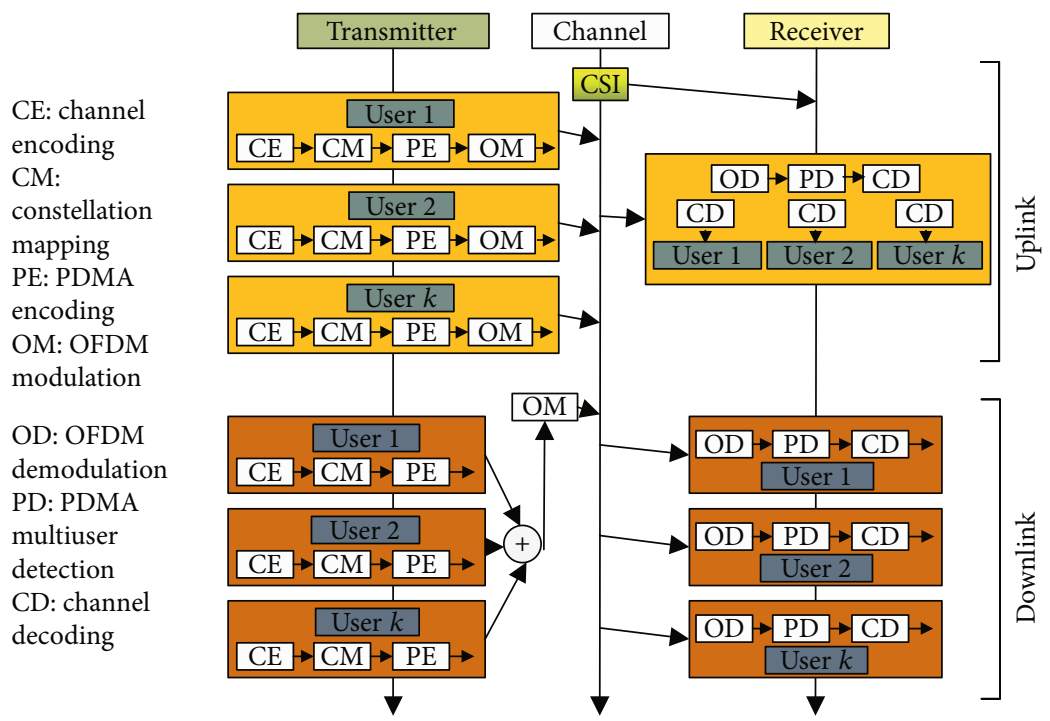

Figure 8: PDMA system model.

Also, throughput at the $i_{\text {th }}$ antenna can be written as

$$
R_{i}=\log _{2}\left(1+\frac{E_{x}\left|w_{i} h_{i}\right|^{2}}{E_{x} \sum_{l \neq i}\left|w_{i} h_{l}\right|+\sigma\left|w_{i}\right|^{2}}\right),
$$

where $E_{x}$ is the transmitted signal energy, $i$ is the number of transmitted antennas, $h_{i}$ is the $i_{\text {th }}$ column of the channel matrix, and $w_{i}$ is the $i_{\text {th }}$ row of the weight matrix. The weight matrix is constructed by using MMSE technique.

3.4. Pattern Division Multiple Access. Pattern Division Multiple Access (PDMA) is well known as an emerging nonorthogonal multiple access technique based on SIC Amenable Multiple Access (SAMA) technology [60]. PDMA utilizes Low Complexity Quasi-ML (LCQ-ML) SIC detection [61] at the reception and holistic/combined scheme of SICAmenable (SIC-A) pattern at the transmission side. An example of the PDMA pattern with resource mapping is shown in Figure 7 [62]. On four resource elements (REs), six users are multiplexed. First of all, the single PDMA pattern is allotted to a single user. All four REs in the cluster are used for userl's data mapping, the first three REs are used for user2, the first and third REs are used for user3, the second and fourth REs are used for user4, the third RE is used for user5, and the fourth RE is used for user6. For all six users, the order of transmission diversity is $4,3,2,2,1$, and 1 [62].

Different users in PDMA are separated at the transmitter through a nonorthogonal character of pattern with various domains, for example, code, space, and power domain. Particularly, at the receiver side, multiple users consist of an irregular diversity degree in order to perform SIC amenable detection. After SIC amenable detection, users can acquire an equivalent diversity degree (Figure 8 [62]). Therefore, in PDMA, steadiness between multiplexing and diversity degree can be achieved [62].

At the receiver side, BS receive signal of " $N$ " resource blocks. The received signal at the $m_{\text {th }}$ antenna of BS is $y_{m}=$ $\left[x_{1, m}, x_{2, m}, x_{3, m}, \cdots, x_{N, m}\right]^{T}$. At the $m_{\text {th }}$ antenna, the received signal of the $n$th resource block can be written as

$$
y_{n, m}=\sum_{k=1}^{K} H_{\mathrm{PDMA}}(n, k) h_{n k, m} \sqrt{P_{n k}} x_{k}+w_{n, m},
$$


TABLE 2: Feature of different NOMA schemes.

\begin{tabular}{|c|c|c|c|}
\hline Type & Advantage & Disadvantage & Key feature \\
\hline PD-NOMA & $\begin{array}{l}\text { (i) Is not affected by apparent near-far } \\
\text { (ii) } 20 \% \text { uplink spectral } \\
\text { efficiency increase } \\
\text { (iii) } 30 \% \text { downlink throughput } \\
\text { increase [64] }\end{array}$ & $\begin{array}{l}\text { (i) High receiver complexity } \\
\text { needs improvement in chip } \\
\text { technology } \\
\text { (ii) Power domain multiplexing } \\
\text { is in the research phase } \\
\text { (iii) SIC increases the system } \\
\text { signalling overhead }\end{array}$ & $\begin{array}{l}\text { (i) For user multiplexing, PD-NOMA utilized } \\
\text { the power domain multiple access } \\
\text { (ii) At receiver, SIC scheme is used } \\
\text { (iii) Take advantage of different channel } \\
\text { conditions }\end{array}$ \\
\hline SCMA & $\begin{array}{l}\text { (i) Three times increase in spectral } \\
\text { efficiency } \\
\text { (ii) } 2.8 \text { times uplink system capacity } \\
\text { upgrade } \\
\text { (iii) } 8 \% \text { and } 5 \% \text { increase in coverage } \\
\text { gain and downlink throughput, } \\
\text { respectively [64] }\end{array}$ & $\begin{array}{l}\text { (i) Optimization and design of } \\
\text { the code are difficult } \\
\text { (ii) Increased interference } \\
\text { between users } \\
\text { (iii) High-dimensional } \\
\text { modulation (HDM) } \\
\text { required }\end{array}$ & $\begin{array}{l}\text { (i) SCMA utilizes sparse spreading sequence, } \\
\text { based on LDS-OFDM } \\
\text { (ii) Spreading with low-density signatures and } \\
\text { bit-to-constellation mapping are combined in } \\
\text { SCMA } \\
\text { (iii) Codebooks are created by multidimensional } \\
\text { constellation. Users' codewords are taken } \\
\text { from codebooks }\end{array}$ \\
\hline MUSA & $\begin{array}{l}\text { (i) Block Error Rate (BLER) is low } \\
\text { (ii) Huge number of users' access is } \\
\text { supported } \\
\text { (iii) Spectral efficiency increased by } 1.5 \\
\text { times [64] }\end{array}$ & $\begin{array}{l}\text { (i) Interuser interference is } \\
\text { increased } \\
\text { (ii) Spread symbol design is } \\
\text { challenging }\end{array}$ & $\begin{array}{l}\text { (i) MUSA is an upgraded scheme of CDMA via } \\
\text { code domain multiplexing } \\
\text { (ii) At the transmitter, MUSA achieved higher } \\
\text { overloading through low-correlation } \\
\text { spreading sequences } \\
\text { (iii) SIC is performed at the receiver side, to } \\
\text { decode superimposed symbols }\end{array}$ \\
\hline PDMA & $\begin{array}{l}\text { (i) } 2-3 \text { times uplink system capacity } \\
\text { increased } \\
\text { (ii) } 1.5 \text { times spectral efficiency increase } \\
\text { in downlink system }\end{array}$ & $\begin{array}{l}\text { (i) The pattern optimization } \\
\text { and design are challenging } \\
\text { (ii) Increase interference } \\
\text { between users }\end{array}$ & $\begin{array}{l}\text { (i) Nonorthogonal patterns are used in PDMA } \\
\text { (ii) Multiplexing is achieved in space domain, } \\
\text { power domain, code domain, and their } \\
\text { composite domain } \\
\text { (iii) Code domain multiplexing is similar to } \\
\text { SCMA } \\
\text { (iv) Low Complexity Quasi-ML SIC detection is } \\
\text { utilized in PDMA }\end{array}$ \\
\hline
\end{tabular}

where " $H_{\mathrm{PDMA}}$ " is the PDMA pattern matrix, " $P_{n k}$ " is the transmitted power of the $k_{\text {th }}$ user at the $n_{\text {th }}$ resource block, and " $x_{k}$ " is the transmitted signal from the $k_{\text {th }}$ user to BS. " $w_{n, m}$ " is complex additive white Gaussian noise at the $n_{\text {th }}$ resource block in $m_{\text {th }}$ receiving antenna.

Using [63], the SINR at the $m_{\text {th }}$ receiving antenna in $n_{\text {th }}$ resource block of the $k_{\text {th }}$ user for the PDMA system can be written as

$$
\operatorname{SINR}_{n k, r}=\frac{P_{n k} H_{\operatorname{PDMA}(n, k)}\left|h_{n k, r}\right|^{2}}{\sigma^{2}+\sum_{j \neq k}^{K} P_{n j} H_{\operatorname{PDMA}(n, j)}\left|h_{n j, r}\right|^{2}} .
$$

Also, throughput for $k_{\text {th }}$ user can be written as

$$
R_{k}=\sum_{r=1}^{N_{r}} \sum_{n=1}^{N} \log _{2}\left(1+\frac{P_{n k} H_{\operatorname{PDMA}(n, k)}\left|h_{n k, r}\right|^{2}}{\sigma^{2}+\sum_{j \neq k}^{K} P_{n j} H_{\operatorname{PDMA}(n, j)}\left|h_{n j, r}\right|^{2}}\right)
$$

where $P_{n k}$ is the power of $k_{\text {th }}$ user in $n_{\text {th }}$ resource block, $H$ is the PDMA pattern matrix, $N_{r}$ indicates the number of receiving antennas, $h_{n k, r}$ is the channel gain coefficient, and $\sigma^{2}$ is the AWGN density. Moreover, Table 2 highlighted the main key features, advantages, and disadvantages of major categories of NOMA schemes.

\section{Summary}

PD-NOMA utilizes nonorthogonal transmission among the users as compared to CDMA and OFDMA. PD-NOMA does not have apparent near-far problem compared to 3G. Similarly, the MAI complications are not challenging in PDNOMA. PD-NOMA has a simple way to respond to multiple links and changing conditions of link by applying Adaptive Modulation and Coding (AMC) particularly in high-speed mobile environments [65]. Therefore, PD-NOMA does not need a highly accurate feedback signal or channel state information (CSI) from the user end. In PD-NOMA, multiple users share the same channel; therefore, spectral efficiency is increased at the unchanged transmission rate compared to $4 \mathrm{G}[66,67]$. In contrast, from a technical implementation aspect, PD-NOMA is still facing several challenges. Initially, implementation needs enhancement in chip technology from signal processing aspect because the nonorthogonal decoder is complex in design. Furthermore, the power domain multiplexing scheme is under the research phase and has a long way to go [64]. Technologies used in different types of nonorthogonal access schemes are presented in Table 3.

As an innovative multiple-access modulation technique, SCMA offered several improvements, for example, multidimensional constellation shaping gain along with benefits of CDMA and LDS. The link-level performance of SCMA in 
TABLE 3: Technology used by NOMA.

\begin{tabular}{lcccc}
\hline Type & PD-NOMA & SCMA & MUSA & PDMA \\
\hline PDM & $\bullet$ & & $\bullet$ & $\bullet$ \\
SIC & $\bullet$ & $\bullet$ & & \\
LDS & & $\bullet$ & & \\
HDM & & $\bullet$ & & \\
MPA & & & $\bullet$ & \\
MCCD & & & & $\bullet$ \\
MLD & & & & \\
\hline
\end{tabular}

highly overloaded scenarios can be achieved by employing channel coding which uses the coding gain and diversity gain. Although the structure of the code is well defined, optimization and design of the code are problematic [64]. To reduce the decoding hurdles of the message passing algorithm, LSD based on a low-complexity decoding algorithm is used in SCMA, in which the LSD only works with signals inside a hypersphere by evading the extensive search for all possible hypotheses.

Uplink access in MUSA utilizes an advanced complex multidomain code structure and multiuser decoding on the basis of SIC. In order to confirm that unlimited reliable access at the same frequency-time slot for multiple users, MUSA makes the procedure of resource allocation simpler in the access scheme. So that MUSA significantly cuts the access time, makes the system implementation simpler, and minimizes energy utilization. MUSA downlink access utilizes superposition symbol expansion and superposition coding scheme, to offer better capacity as compared to downlink transmission provided by the OMA. Also, uplink access in MUSA offers to decrease the energy consumption and make the implementation of user terminal simpler which is the same as MUSA downlink [64].

PDMA can increase the performance of the spectrum utilization for the downlink system by 1.5 times and increases capacity in the uplink system by 2-3 times [68]. To improve the security in the PDMA system, constellation scrambling with MP-WFRFT is utilized. To avoid error propagation, MMSE channel decoding and detection-based SIC iterative processing is used in the PDMA system. Cooperative PDMA is used with forward relay and half-duplex decode in order to further enlarge the coverage area and improve transmission reliability. On the other side, PDMA needs to encounter some important challenges to be resolved in upcoming applications. These include designing simpler receivers, design patterns at the transmission end to discriminate users without difficulty, and combine MIMO with PDMA in order to develop space domain coding design, etc.

\section{Discussion}

System sum rate performance versus the total number of users of PD-NOMA, SCMA, MUSA, and PDMA is illustrated in Figure 9 from [69]. SCMA has been confirmed by theory and in lab tests that SCMA has a better sum rate among all major four categories while the complexity of
SCMA is bigger than the PD-NOMA due to the code domain. SCMA is capable of achieving coding gains and improved shaping. SCMA allows a fixed number of resource blocks to each user while PDMA allows a changeable number of resource blocks to each user, since, in PDMA, the user data rate is different, which results in degradation of the system sum rate. PD-NOMA and MUSA both utilized SIC, but PD-NOMA utilized power domain multiple access and MUSA utilized a special spread sequence to spread the user's data symbols. Therefore, MUSA has a better sum rate than PD-NOMA.

A comparison of the average aggregate energy efficiency of PD-NOMA and SCMA schemes against the number of its users is illustrated in Figure 10 from [70]. SCMA outperforms its counterparts due to nonorthogonality with high overloading. Therefore, in SCMA, further access of users is achieved with low energy consumption. PD-NOMA also utilized nonorthogonal access; more users can be employed on less numbers of resources, but due to power domain access, overloading cannot be achieved. On the other hand, OFDMA underperforms because OFDMA is an orthogonal scheme in which users are restricted by orthogonal resources.

Figure 11 illustrated the bit error rate of PD-NOMA, SCMA, PDMA, and MUSA uplink systems in the Rayleigh fading channel from [71]. For performance comparison of the PDMA and SCMA, the same factor graph is used with QPSK modulation. The number of orthogonal resources is 4 , and the number of symbols which are transmitted is 6 . Therefore, the resulting overloading factor becomes $150 \%$. With the help of [72], the codebooks are designed in SCMA. Pseudorandom sequences whose image and real values are obtained from set $\{-1,0,1\}$ are used to generate spreading sequences for MUSA, and nonorthogonal patterns are designed accordingly [73] for PDMA.

The SCMA uplink system has high-quality BER performance among all code domains, as shown in Figure 11. However, the BER performance of MUSA and PDMA is very similar and lesser than SCMA. The effect of error propagation of the SIC receiver on the system performance is the main reason for performance degradation of MUSA and PDMA. Nevertheless, when PDMA utilized the unchanged factor graph as used in SCMA, SCMA still has better BER performance than PDMA. The reason for this performance improvement is because of the near-optimal strategy of sparse codewords. On the other hand, the code domain NOMA scheme achieves a better system sum rate than the power domain NOMA scheme; therefore, PD-NOMA managed poor performance among all.

\section{Recent Development}

The race for developing $5 \mathrm{G}$ technology has integrated NOMA with different communication technologies, such as NOMA-based communication for the Tactile Internet, NOMA for D2D communication, cognitive radio nonorthogonal multiple access, and SWIPT-NOMA-based HetNets. A brief review of recent developments in NOMA is as follows.

The authors in [74] presented NOMA-based applicationspecific communication for the Tactile Internet, by which 


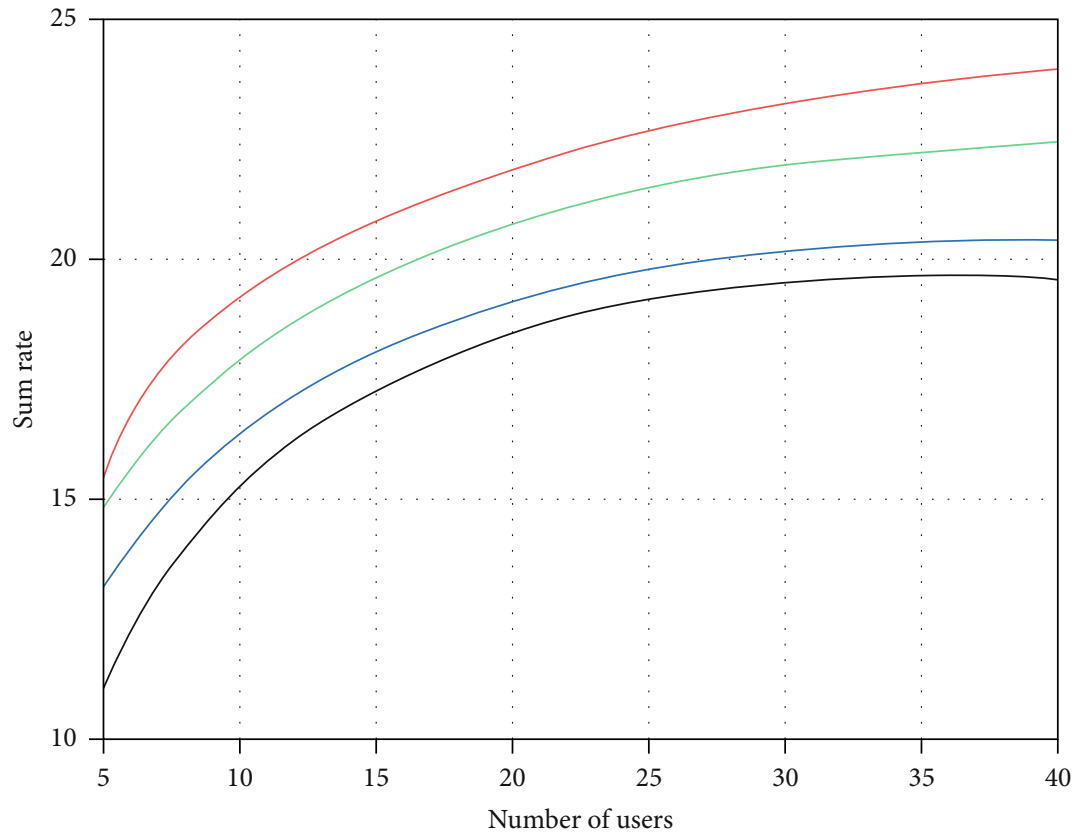

Sum rate

- PD-NOMA

- MUSA

- PDMA

- SCMA

Figure 9: Sum rate of different non-orthogonal schemes.

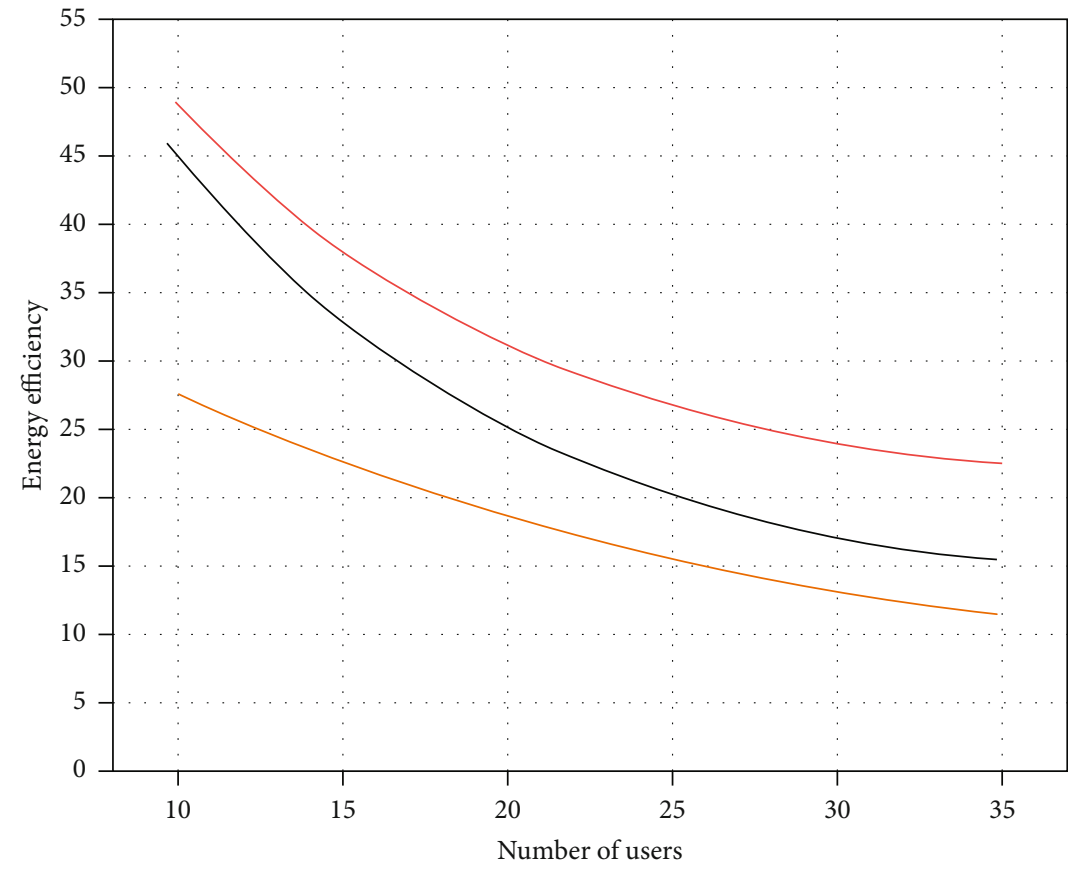

Energy efficiency

$$
\begin{aligned}
& \text { — PD-NOMA } \\
& \text { — OFDMA } \\
& \text { — SCMA }
\end{aligned}
$$

FIGURE 10: Energy efficiency of different orthogonal and non-orthogonal schemes. 


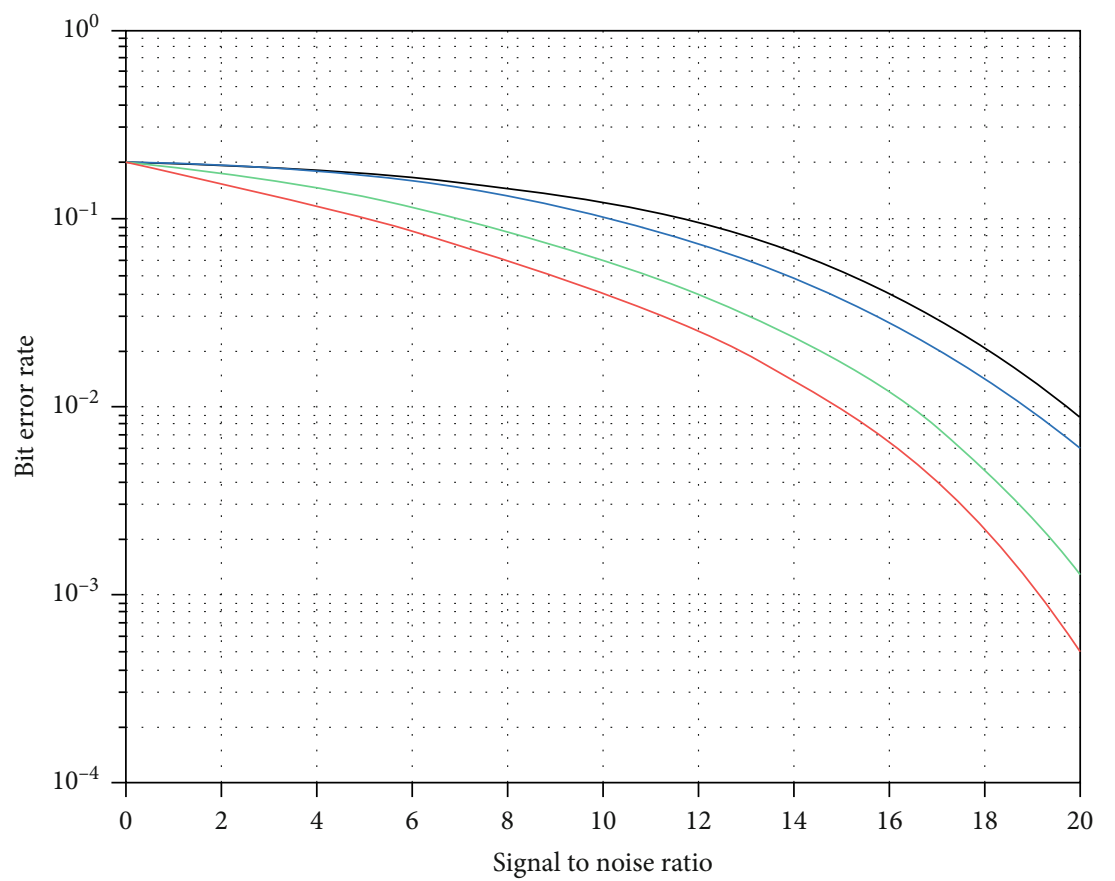

BER vs. SNR

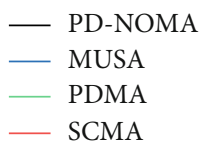

FIGURE 11: BER performance of different non-orthogonal schemes.

heterogeneity can be achieved in 5G networks. Tactile Internet allows nonorthogonal resource sharing from a pool of massive machine-type communications (mMTC), ultrareliable low latency communications (URLLC), enhanced mobile broadband (eMBB), and critical machine-type communication (cMTC) devices to a shared the same base station. The authors in [74] summarized many different types of NOMA and their appropriateness for low latency Tactile Internet-based applications. Additionally, the authors in [74] presented a sample case of a healthcare-based network and explained how in the healthcare domain NOMA-based architecture can be utilized for low latency networks.

In [75], the authors used NOMA at the D2D transmitter to improve the spectral efficiency of the network. The authors proposed in [75] the Tactile Internet Driven Delay Assessment for D2D communication (DIYA) scheme to resolve the issue of interference and delay from the neighboring nodes in two-hop transmission. In the first phase at relays (intermediate nodes), a full duplex communication is used for the first and second hop transmission concurrently, at the same time interval. Afterward, at D2D transmitter transmission rate is improved using Tactile Internet-based communication. In the second phase, to reduce the cochannel interference and increase the throughput of the cell edge users, pricing-based $3 \mathrm{D}$ matching is proposed by authors. Furthermore, authors in [75] used successive convex approximation (SCA) with less complexity in order to optimize the power of the D2D transmitter. SCA converts the nonconvex optimization problem of power control and subchannel allocation into the convex problem.

In [76], to improve the sum rate of the femtocell users, researchers proposed a joint power control and channel allocation algorithm by utilizing cognitive radio nonorthogonal multiple access (CR-NOMA) at the femtobase station. The authors used the channel gain difference among weak and strong users' pairs in the proposed algorithm. This reduces the interference between NOMA users and improves channel utilization. Furthermore, to provide the QoS for weak users, the authors differentiated the odd and even numbers of users in a femtocell. The aforementioned scheme, OMA, is utilized to obtain a preset data rate by a greedy channel allocation algorithm.

The authors in [77] presented a subchannel assignment scheme for SWIPT-NOMA-based HetNets with imperfect CSI for the downlink system. Furthermore, the many-tomany matching theory is presented by authors to formulate the subchannel assignment. Considering imperfect CSI, the authors in [77] presented the energy-efficient subchannel assignment as a nonconvex probabilistic optimization problem. The many-to-many matching theory is utilized by authors to deal with this problem. The authors used SWIPT and NOMA with macrousers and pico-/femtobase station, in which multiple users served by NOMA simultaneously and SWIPT harvest energy from the radio frequency signals. Both techniques increase the energy efficiency of the network. 
In [78], to maximize the sum rate and spectral efficiency of femtousers with guaranteed QoS, the authors investigated the NOMA transmission with $5 \mathrm{G}$ enabled cognitive femtocell. To reduce the NOMA interference among multiple femtousers, a pairing algorithm between weak and strong users has been presented by authors. The authors also calculated the sum rate for an even/odd number of femtousers in order to achieve a higher data rate.

\section{Research Challenges}

In this article, we investigated major categories of NOMA, contributions of NOMA in enabling the 5G network, integration with different communication technologies, and recent research trends. Conversely, there are still many challenges which should be solved further to improve the performance of NOMA systems. We present various significant challenges of NOMA and specify potential research.

7.1. Imperfect SIC Cancellation. In practical circumstances during SIC processing, some residual interference left; the successive interference cancellation is mostly imperfect. Therefore, in theoretical analysis, we have to consider this imperfect cancellation aspect. Furthermore, error propagation in SIC is also a major problem. This indicates that when the higher-order user has been decoded erroneously, the error will sequentially propagate to lower-order users.

7.2. Imperfect CSI. The current research works on NOMA presume a perfect CSI to implement multiuser interference cancellation at the user receiver or resource allocation at BS. However, perfect CSI is impossible in practical scenarios. Therefore, real-time NOMA systems work with channel estimation errors. In theoretical analysis of NOMA, there is a need to consider channel estimation errors and imperfect CSI.

7.3. Design of Spreading Sequences or Codebooks. In SCMA, codebook design is still an issue particularly for outsized higher-dimensional codebooks. For further performance improvement of SCMA, the joint design of the factor graph matrix and constellation construction is required. For this, advance multidimension constellation is needed. Furthermore, to improve link adaptation, the design scheme for the case that all the overloaded users have different codebook sizes (transmission rate) needs to be investigated. Moreover, to determine the performance and capacity under practical scenarios, researchers have to consider error propagation in codebook allocation in theoretical analysis.

7.4. Receiver Complexity. As compared to OMA schemes, in NOMA, SIC needs additional implementation complexity, because the SIC receiver has to detect and cancel other users' signals prior to detecting its own signal. Moreover, as the number of users in the cell increases, the receiving complexity also increases. Therefore, a high-performance nonlinear detection algorithm is required at each stage of SIC for error-free propagation.
7.5. Heterogeneous Networks. A wireless network containing nodes with different coverage sizes and transmission powers is known as a heterogeneous network (HetNet). The HetNet has capability in terms of coverage and capacity with reduced energy consumption for future wireless networks. Real-time NOMA allows sharing of resources for different types of networks. To improve system throughput of heterogeneous networks, heterogeneous collaborative communication schemes with NOMA can be investigated.

7.6. Further Challenges. Several further challenges of NOMA systems must also be addressed, including signal design and channel estimation, maintaining system scalability, for multicarrier NOMA the reduction of the PAPR, the difficulties of channel-quality feedback design, and flexible configuration of multiple access schemes. It is accepted by researchers that by addressing these challenges NOMA will further improve.

7.7. Future Trends. As the expected new round of developments, NOMA has received huge attention and active input from researchers, and its development is very rapid. Some future research trends are NOMA in large-scale heterogeneous networks, full-/half-duplex user relaying in NOMA systems, NOMA for wireless powered IoT networks, NOMA-based massive MTC networks, adaptive NOMA/OMA mode-switching, NOMA systems over $\kappa-\mu$ shadowed fading channels, in large-scale underlay cognitive radio networks, and NOMA with spatial modulation.

\section{Conclusion}

Currently, nonorthogonal awareness has been significantly useful in the modern developments in the 5G multiple access scheme. Herein, favorable nonorthogonal multiple access technique for $5 \mathrm{G}$ mobile communications is reassessed and compared on the basis of their advantages, disadvantages, and key features and their future development. Furthermore, we considered the performance of significant NOMA schemes, i.e., PD-NOMA, SCMA, MUSA, and PDMA in Rayleigh fading channels. This comparison research reveals the performance of different NOMA schemes. With the indepth review of their basic working principle, system model, and performance, 5G key multiple access technique will be progressively understood, enabling us to arrive at the essential stage of formulation and standardization.

\section{Conflicts of Interest}

The authors declared that there is no conflict of interest regarding the publication of this paper.

\section{Acknowledgments}

This work would be funded by authors.

\section{References}

[1] L. Dai, B. Wang, Z. Ding, Z. Wang, S. Chen, and L. Hanzo, "A survey of non-orthogonal multiple access for 5G," IEEE 
Communications Surveys \& Tutorials, vol. 20, no. 3, pp. 22942323, 2018.

[2] Y. Chen, A. Bayesteh, Y. Wu et al., "Toward the standardization of non-orthogonal multiple access for next generation wireless networks," IEEE Communications Magazine, vol. 56, no. 3, pp. 19-27, 2018.

[3] A. Benjebbour, K. Saito, and Y. Kishiyama, "Experimental trials on non-orthogonal multiple access," in In Multiple Access Techniques for $5 G$ Wireless Networks and Beyond, pp. 587607, Springer, 2019.

[4] H. Nikopour and H. Baligh, "Sparse code multiple access," in 2013 IEEE 24th Annual International Symposium on Personal, Indoor, and Mobile Radio Communications (PIMRC), pp. 332336, London, UK, 2013.

[5] Z. Yuan, G. Yu, and W. Li, "Multi-user shared access for 5G," Telecommunication Network Technology, vol. 5, no. 5, pp. 2830, 2015.

[6] R. Hoshyar, F. P. Wathan, and R. Tafazolli, "Novel low-density signature for synchronous CDMA systems over AWGN channel," IEEE Transactions on Signal Processing, vol. 56, no. 4, pp. 1616-1626, 2008.

[7] X. Dai, Z. Zhang, B. Bai, S. Chen, and S. Sun, "Pattern division multiple access: a new multiple access technology for $5 \mathrm{G}$," IEEE Wireless Communications, vol. 25, no. 2, pp. 54-60, 2018.

[8] Z. Wu, K. Lu, C. Jiang, and X. Shao, "Comprehensive study and comparison on 5G NOMA schemes," IEEE Access, vol. 6, pp. 18511-18519, 2018.

[9] L. Zhang, M. Xiao, G. Wu, M. Alam, Y. C. Liang, and S. Li, “A survey of advanced techniques for spectrum sharing in $5 \mathrm{G}$ networks," IEEE Wireless Communications, vol. 24, no. 5, pp. 44$51,2017$.

[10] Y. Wang, B. Ren, S. Sun, S. Kang, and X. Yue, "Analysis of nonorthogonal multiple access for 5G," China Communications, vol. 13, no. 2, pp. 52-66, 2016.

[11] Y. El Gholb, N. El Amrani, E. Idrissi, and H. Ghennioui, "5G: an idea whose time has come," International Journal of Scientific and Engineering Research, vol. 8, no. 3, 2017.

[12] A. P. Singh, S. Nigam, and N. K. Gupta, "A study of next generation wireless network 6G," International Journal of Innovative Research in Computer and Communication Engineering, vol. 4, no. 1, 2007.

[13] A. Benjebbour, K. Saito, A. Li, Y. Kishiyama, and T. Nakamura, "Non-orthogonal multiple access (NOMA): concept, performance evaluation and experimental trials," in 2015 International Conference on Wireless Networks and Mobile Communications (WINCOM), pp. 1-6, Marrakech, Morocco, 2015.

[14] J. Kim, J. Lee, D. Kim, and Y. Choi, "System-level performance evaluation for non-orthogonal multiple access in coordinated direct and relay transmission," in 2017 International Conference on Information and Communication Technology Convergence (ICTC), pp. 1296-1298, Jeju, South Korea, 2017.

[15] Z. Ding, P. Fan, and H. V. Poor, "Impact of user pairing on 5G nonorthogonal multiple-access downlink transmissions," IEEE Transactions on Vehicular Technology, vol. 65, no. 8, pp. 6010-6023, 2016.

[16] H. Xing, Y. Liu, A. Nallanathan, Z. Ding, and H. V. Poor, "Optimal throughput fairness tradeoffs for downlink nonorthogonal multiple access over fading channels," IEEE Transactions on Wireless Communications, vol. 17, no. 6, pp. 35563571, 2018.
[17] S. Arzykulov, T. A. Tsiftsis, G. Nauryzbayev, and M. Abdallah, "Outage performance of cooperative underlay CR-NOMA with imperfect CSI," IEEE Communications Letters, vol. 23, no. 1, pp. 176-179, 2019.

[18] P. Yang, Y. Xiao, M. Xiao, and Z. Ma, "NOMA-Aided precoded spatial modulation for downlink MIMO transmissions," IEEE Journal of Selected Topics in Signal Processing, vol. 13, no. 3, pp. 729-738, 2019.

[19] L. Song, S. Li, and Y. Sun, "Power allocation for full-duplex NOMA relaying based underlay D2D communications," KSII Transactions on Internet and Information Systems, vol. 13, no. 1, pp. 16-33, 2019.

[20] L. Anxin, A. Benjebbour, X. Chen, H. Jiang, and H. Kayama, "Uplink non-orthogonal multiple access (NOMA) with single-carrier frequency division multiple access (SC-FDMA) for 5G systems," IEICE Transactions on Communications., vol. E98.B, no. 8, pp. 1426-1435, 2015.

[21] B. Xia, J. Wang, K. Xiao, Y. Gao, Y. Yao, and S. Ma, “Outage performance analysis for the advanced SIC receiver in wireless NOMA systems," IEEE Transactions on Vehicular Technology, vol. 67, no. 7, pp. 6711-6715, 2018.

[22] S. Riaz, J. Kim, and U. Park, "Evolutionary game theory-based power control for uplink NOMA," KSII Transactions on Internet and Information Systems, vol. 12, no. 6, pp. 2697-2710, 2018.

[23] B. Makki, K. Chitti, A. Behravan, and M. S. Alouini, "A survey of NOMA: current status and open research challenges," IEEE Open Journal of the Communications Society, vol. 1, pp. 179189,2020

[24] H. Tabassum, E. Hossain, and M. J. Hossain, "Modeling and analysis of uplink non-orthogonal multiple access (NOMA) in large-scale cellular networks using Poisson cluster processes," IEEE Transactions on Communications, vol. 65, no. 8, pp. 3555-3570, 2017.

[25] P. Merias and J. M. Meredith, Study on non-orthogonal multiple access (NOMA) for NR, 3GPP, Sophia Antipolis, France, 2018, Rep. TR 38.812.

[26] Z. Ding, M. Peng, and H. V. Poor, "Cooperative nonorthogonal multiple access in $5 \mathrm{G}$ systems," IEEE Communications Letters, vol. 19, no. 8, pp. 1462-1465, 2015.

[27] S. Timotheou and I. Krikidis, "Fairness for non-orthogonal multiple access in 5G systems," IEEE Signal Processing Letters, vol. 22, no. 10, pp. 1647-1651, 2015.

[28] Z. Ding, F. Adachi, and H. V. Poor, "The application of MIMO to non-orthogonal multiple access," IEEE Transactions on Wireless Communications, vol. 15, no. 1, pp. 537552, 2016.

[29] Y. Sun, D. W. K. Ng, Z. Ding, and R. Schober, "Optimal joint power and subcarrier allocation for full-duplex multicarrier non-orthogonal multiple access systems," IEEE Transactions on Communications, vol. 65, no. 3, pp. 1077-1091, 2017.

[30] F. Fang, H. Zhang, J. Cheng, and V. C. M. Leung, "Energy-efficient resource allocation for downlink non-orthogonal multiple access network," IEEE Transactions on Communications, vol. 64, no. 9, pp. 3722-3732, 2016.

[31] Y. Wu, S. Zhang, and Y. Chen, "Iterative multiuser receiver in sparse code multiple access systems," in 2015 IEEE International Conference on Communications (ICC), pp. 2918-2923, London, UK, 2015.

[32] C. Dong, G. Gao, K. Niu, and J. Lin, "An efficient SCMA codebook optimization algorithm based on mutual information 
maximization," Wireless Communications and Mobile Computing, vol. 2018, 13 pages, 2018.

[33] Y. Du, B. Dong, Z. Chen, J. Fang, and L. Yang, "Shuffled multiuser detection schemes for uplink sparse code multiple access systems," IEEE Communications Letters, vol. 20, no. 6, pp. 1231-1234, 2016.

[34] F. Wei and W. Chen, "Low complexity iterative receiver design for sparse code multiple access," IEEE Transactions on Communications, vol. 65, no. 2, pp. 621-634, 2017.

[35] J. Chen, Z. Zhang, S. He, J. Hu, and G. E. Sobelman, "Sparse code multiple access decoding based on a Monte Carlo Markov chain method," IEEE Signal Processing Letters, vol. 23, no. 5, pp. 639-643, 2016.

[36] M. Moltafet, N. Mokari, M. R. Javan, H. Saeedi, and H. PishroNik, "A new multiple access technique for 5G: power domain sparse code multiple access (PSMA)," IEEE Access, vol. 6, pp. 747-759, 2018.

[37] Z. Yuan, C. Yan, Y. Yuan, and W. Li, "Blind Multiple User Detection for Grant-Free MUSA without Reference Signal," in 2017 IEEE 86th Vehicular Technology Conference (VTCFall), pp. 1-5, Toronto, ON, USA, 2017.

[38] Y. Xu, G. Wang, L. Zheng, R. Liu, and D. Zhao, "BER performance evaluation of downlink MUSA over Rayleigh fading channel," in International Conference on Machine Learning and Intelligent Communications, pp. 85-94, Springer, 2017.

[39] 3GPP Document R1-166404, "Receiver details and link performance for MUSA," in 3GPP TSG RAN WG1 Meeting No. 86, Gothenburg, Sweden, 2016.

[40] N. Ye, H. Han, L. Zhao, and A. H. Wang, "Uplink nonorthogonal multiple access technologies toward 5G: a survey," Wireless Communications and Mobile Computing, vol. 2018, 26 pages, 2018.

[41] W. Tang, S. Kang, and B. Ren, "Performance analysis of cooperative pattern division multiple access (co-PDMA) in uplink network," IEEE Access, vol. 5, pp. 3860-3868, 2017.

[42] B. Ren, X. Yue, W. Tang et al., "Advanced IDD receiver for PDMA uplink system," in 2016 IEEE/CIC International Conference on Communications in China (ICCC), pp. 1-6, Chengdu, China, 2016.

[43] D. Kong, J. Zeng, X. Su, L. Rong, and X. Xu, "Multiuser detection algorithm for PDMA uplink system based on SIC and MMSE," in 2016 IEEE/CIC International Conference on Communications in China (ICCC), pp. 1-5, Chengdu, China, 2016.

[44] J. Zeng, B. Liu, and X. Su, "Joint pattern assignment and power allocation in PDMA," in 2017 IEEE 86th Vehicular Technology Conference (VTC-Fall), pp. 1-5, Toronto, ON, USA, 2017.

[45] B. Ren, Y. Wang, X. Dai, K. Niu, and W. Tang, "Pattern matrix design of PDMA for 5G UL applications," China Communications, vol. 13, Supplement2, pp. 159-173, 2016.

[46] G. Mazzini, "Power division multiple access," in ICUPC '98. IEEE 1998 International Conference on Universal Personal Communications. Conference Proceedings (Cat. No.98TH8384), pp. 543-546, Florence, Italy, Italy, 1998.

[47] Y. Saito, Y. Kishiyama, A. Benjebbour, T. Nakamura, A. Li, and K. Higuchi, "Non-orthogonal multiple access (NOMA) for cellular future radio access. Vehicular Technology Conference (VTC Spring)," in 2013 IEEE 77th Vehicular Technology Conference (VTC Spring), pp. 1-5, Dresden, Germany, 2013.

[48] A. Benjebbour, Y. Saito, Y. Kishiyama, A. Li, A. Harada, and T. Nakamura, "Concept and practical considerations of nonorthogonal multiple access (NOMA) for future radio access," in 2013 International Symposium on Intelligent Signal Processing and Communication Systems, pp. 770-774, Naha, Japan, 2013.

[49] X. Chen, A. Beiijebbour, A. Li, H. Jiang, and H. Kayama, "Consideration on successive interference canceller (SIC) receiver at cell-edge users for non-orthogonal multiple access (NOMA) with SU-MIMO," in 2015 IEEE 26th Annual International Symposium on Personal, Indoor, and Mobile Radio Communications (PIMRC), pp. 522-526, Hong Kong, China, 2015.

[50] G. Gui, H. Sari, and E. Biglieri, "A new definition of fairness for non-orthogonal multiple access," in IEEE Communications Letters, vol. 23, no. 7, pp. 1267-1271, 2019.

[51] B. Di, L. Song, and Y. Li, "Radio resource allocation for uplink sparse code multiple access (SCMA) networks using matching game," in 2016 IEEE International Conference on Communications (ICC), pp. 1-6, Kuala Lumpur, 2016.

[52] M. Hussain and H. Rasheed, "A Computational Power Allocation Scheme for Fair NOMA Downlink System," Journal of Information Communication Technologies and Robotic Applications (JICTRA), vol. 9, no. 1, pp. 73-79, 2018.

[53] S. Zhang, K. Xiao, B. Xiao et al., "A capacity-based codebook design method for sparse code multiple access systems," in 2016 8th International Conference on Wireless Communications \& Signal Processing (WCSP),, pp. 1-5, Yangzhou, China, 2016.

[54] Y. Zhou, H. Luo, R. Li, and J. Wang, "A dynamic states reduction message passing algorithm for sparse code multiple access," in 2016 Wireless Telecommunications Symposium (WTS), pp. 1-5, London, UK, 2016.

[55] K. Au, L. Zhang, H. Nikopour et al., "Uplink contention based scma for 5G radio access," in 2014 IEEE Globecom Workshops (GC Wkshps), pp. 900-905, 2014.

[56] Z. Yang, J. Cui, X. Lei, Z. Ding, P. Fan, and D. Chen, "Impact of factor graph on average sum rate for uplink sparse code multiple access systems," IEEE Access, vol. 4, pp. 6585-6590, 2016.

[57] K. Hyukjoon, L. Jungwon, and K. Inyup, "Successive interference cancellation via rank-reduced maximum a posteriori detection," Communications, IEEE Transactions on, vol. 61, no. 2, pp. 628-637, 2013.

[58] Z. Yuan, G. Yu, W. Li, Y. Yuan, X. Wang, and J. Xu, "Multiuser shared access for Internet of things," in 2016 IEEE 83rd Vehicular Technology Conference (VTC Spring), pp. 1-5, Nanjing, China.

[59] E. M. Eid, M. M. Fouda, A. S. T. Eldien, and M. M. Tantawy, "Performance analysis of MUSA with different spreading codes using ordered SIC methods," in 2017 12th International Conference on Computer Engineering and Systems (ICCES), pp. 101-106, Cairo, Egypt, 2017.

[60] X. Dai, "Successive interference cancellation amenable space-time codes with good multiplexing-diversity Tradeoffs," Wireless Personal Communication, vol. 55, no. 4, pp. 645-654, 2010.

[61] X. Dai, S. Sun, and Y. Wang, "Reduced-complexity (quasi-) maximum- likelihood detectors with no performance degradation for S-QAM modulated MIMO systems," Wireless Personal Communication, vol. 66, no. 4, pp. 613-627, 2012.

[62] S. Chen, B. Ren, Q. Gao, S. Kang, S. Sun, and K. Niu, "Pattern division multiple access (PDMA) - a novel non-orthogonal multiple access for 5G radio networks," IEEE Transactions on Vehicular Technology, vol. 66, no. 4, 2019.

[63] J. Zeng, D. Kong, X. Su, L. Rong, and X. Xu, "On the performance of pattern division multiple access in $5 \mathrm{G}$ systems," in 
2016 8th International Conference on Wireless Communications \& Signal Processing (WCSP), pp. 1-5, Yangzhou, China, 2016.

[64] Y. Tao, L. Liu, S. Liu, and Z. Zhang, "A survey: several technologies of non-orthogonal transmission for 5G," China Communications, vol. 12, no. 10, pp. 1-15, 2015.

[65] Q. Liu, S. Zhou, and G. B. Giannakis, "Cross-layer combining of adaptive modulation and coding with truncated ARQ over wireless links," IEEE Transactions on Wireless Communications, vol. 3, no. 5, pp. 1746-1755, 2004.

[66] Y. Saito, A. Benjebbour, Y. Kishiyama, and T. Nakamura, "System-level performance evaluation of downlink nonorthogonal multiple access (NOMA)," in 2013 IEEE 24th Annual International Symposium on Personal, Indoor, and Mobile Radio Communications (PIMRC)., pp. 611-615, London, UK, 2013.

[67] M. Al-Imari, P. Xiao, M. A. Imran, and R. Tafazolli, "Uplink non-orthogonal multiple access for 5G wireless networks," in 2014 11th International Symposium on Wireless Communications Systems (ISWCS), pp. 781-785, Barcelona, Spain, 2014.

[68] M. Hussain and H. Rasheed, "Performance of Orthogonal Beamforming with NOMA for Smart Grid Communication in the Presence of Impulsive Noise," Arabian Journal for Science and Engineering (AJSE), vol. 45, pp. 6331-6345, 2020.

[69] M. Moltafet, N. M. Yamchi, M. R. Javan, and P. Azmi, "Comparison study between PD-NOMA and SCMA," IEEE Transactions on Vehicular Technology, vol. 67, no. 2, pp. 18301834, 2018.

[70] Y. Dong, L. Qiu, and X. Liang, "Energy efficiency maximization for uplink SCMA system using CCPSO," in 2016 IEEE Globecom Workshops (GC Wkshps), pp. 1-5, Washington, DC, USA, 2016.

[71] B. Wang, K. Wang, Z. Lu, T. Xie, and J. Quan, "Comparison study of non-orthogonal multiple access schemes for 5G," in 2015 IEEE International Symposium on Broadband Multimedia Systems and Broadcasting, pp. 1-5, Ghent, Belguim, 2015.

[72] M. Taherzadeh, H. Nikopour, A. Bayesteh, and H. Baligh, "SCMA codebook design," in 2014 IEEE 80th Vehicular Technology Conference (VTC2014-Fall),, pp. 1-5, Vancouver, BC, Canada, 2014.

[73] X. Dai, S. Chen, S. Sun et al., "Successive interference cancelation amenable multiple access (SAMA) for future wireless communications," in 2014 IEEE International Conference on Communication Systems, pp. 222-226, Macau, China, 2014.

[74] I. Budhiraja, S. Tyagi, S. Tanwar, N. Kumar, and J. J. P. C. Rodrigues, "Tactile internet for smart communities in 5G: an insight for NOMA-based solutions," IEEE Transactions on Industrial Informatics, vol. 15, no. 5, pp. 3104-3112, 2019.

[75] I. Budhiraja, S. Tyagi, S. Tanwar, N. Kumar, and J. J. P. C. Rodrigues, "DIYA: tactile internet driven delay assessment NOMA-based scheme for D2D communication," IEEE Transactions on Industrial Informatics, vol. 15, no. 12, pp. 63546366, 2019.

[76] I. Budhiraja, S. Tyagi, S. Tanwar, N. Kumar, and M. Guizani, "Cross layer NOMA interference mitigation for femtocell users in 5G environment," IEEE Transactions on Vehicular Technology, vol. 68, no. 5, pp. 4721-4733, 2019.
[77] I. Budhiraja, S. Tyagi, S. Tanwar, N. Kumar, and N. Guizani, "Subchannel assignment for SWIPT-NOMA based HetNet with imperfect channel state information," in 2019 15th International Wireless Communications \& Mobile Computing Conference (IWCMC), pp. 842-847, Tangier, Morocco, 2019.

[78] I. Budhiraja, S. Tyagi, S. Tanwar, N. Kumar, and M. Guizani, "CR-NOMA based interference mitigation scheme for $5 \mathrm{G}$ femtocells users," in 2018 IEEE Global Communications Conference (GLOBECOM), pp. 1-6, Abu Dhabi, United Arab Emirates, 2018. 